\title{
Contextual action recognition and target localisation with active allocation of attention on a humanoid robot
}

\author{
Dimitri Ognibene, Eris Chinellato, Miguel Sarabia \& Yiannis \\ Demiris \\ Imperial College London, South Kensington Campus, London SW7 2AZ, UK \\ E-mail: \\ $\{$ d.ognibene, e.chinellato, miguel.sarabia,y.demiris\}@imperial.ac.uk
}

\begin{abstract}
Exploratory gaze movements are fundamental for gathering the most relevant information regarding the partner during social interactions. Inspired by the cognitive mechanisms underlying human social behaviour, we have designed and implemented a system for dynamic attention allocation which is able to actively control gaze movements during a visual action recognition task exploiting its own action execution predictions. Our humanoid robot is able, during the observation of a partner's reaching movement, to contextually estimate the goal position of the partner's hand and the location in space of the candidate targets. This is done while actively gazing around the environment, with the purpose of optimising the gathering of information relevant for the task. Experimental results on a simulated environment show that active gaze control, based on the internal simulation of actions, provides a relevant advantage with respect to other action perception approaches, both in terms of estimation precision and of time required to recognise and action. Moreover, our model reproduces and extends some experimental results on human attention during action perception.
\end{abstract}




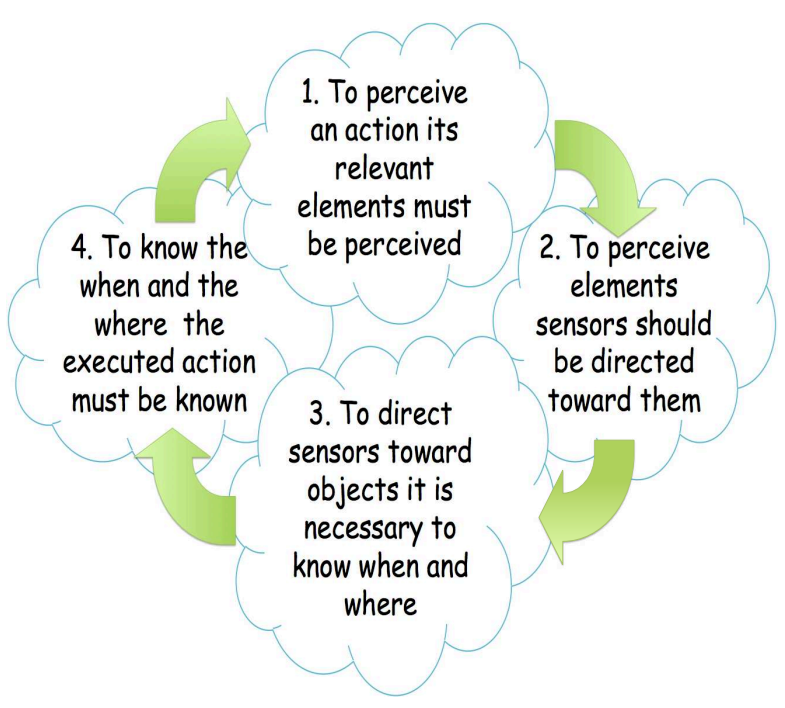

Figure 1. The circular problem of directing sensors to perceive events with multiple candidate participant objects.

\section{Introduction}

Biological organisms make extensive use of task-specific representations, computations and attention control skills to face dynamic, stochastic and partially unknown environments with their limited knowledge and computational resources.

Humans actively search for task-relevant stimuli rather than passively processing sensory information or executing a complete reconstruction of the world state. Converging evidence shows that task-specific information plays a key role in gaze control, and that the influence of low-level image statistics (bottom-up saliency [8]) is instead minor $[28,16]$. Moreover, humans retain and process only the information that is useful for the task, and aim at improving the gathering of such information [22].

In computer vision, the introduction of active vision $[2,1]$ was a fundamental step towards overcoming the limits of the classical vision paradigm as formulated by Marr [12]. Nevertheless, the perception of dynamic events still poses fundamental problems, such as the timely detection of the relevant elements, and the recognition of the discriminant dynamics. Event perception requires, both for the recognition and for the direction of the gaze, the integration through time of the acquired information. Event perception has wide behavioural relevance and in most of these conditions, e.g. a goal keeper during a penalty kick, it is extremely important not only to recognise an event, but to anticipate it, in order to prepare and execute a proper action plan in response [27].

In this context, the temporal dimension has additional importance because a wrong timing in attentional control may lead to overlooking important clues for event recognition, e.g. like in a magic show, when the public get distracted and cannot recognise the trick of the wizard. To be more specific, directing attention to perceive events with multiple candidate participating objects is a kind of circular problem (see 
figure 1), because: a) recognising the event requires to perceive the temporal changes, interactions and trajectories of the objects participating in the event; b) perceiving the objects requires to properly direct the sensors during the event; c) to direct the sensors, both the actual set of candidate objects and their trajectories should be known. The latter closes the loop by depending on the event itself. This paper proposes a bioinspired method to solve some instances of this problem and compares this method to other approaches.

An archetypal and behaviourally relevant example of event perception is the recognition of an action executed by another agent. The segmentation of other agents behaviours in terms of goal-directed actions, and the recognition of the goals permit the production of longer predictions [26]. It is thus essential for a majority of human social behaviours [25]. In order to deliver a behavioural advantage - and to allow for timely action selection - the target (e.g apple) and the end effector (e.g. hand) of an action should be predicted in advance, notwithstanding the limited perceptual and computational resources of the observer, and her knowledge of the environment, which is never optimal, due to occlusions and inner visual complexity.

Another important issue in action perception is the knowledge demand necessary to recognise the different actions. In this regard we follow the simulation theory of action perception (see [14] for a review), which allows the reuse of the same knowledge and the same mechanism to perceive actions by simulating them in the motor system. An additional advantage of using a simulative, and thus predictive, approach is that it creates a richer informational context which can support attention control.

Starting from the simulation theory of mind point of view for action perception, we manage attention allocation in an active way, according to the predicted plausibility of candidate actions and possible targets. For a given action, the information that the attention system extracts during action observation is the value of the corresponding variables that an inverse model would control if it was executing that same action. For example, the inverse model for executing an arm movement will request the state of the arm when used in perception mode. This novel approach provides a principled way for supplying top-down signals to the attention system, which is to be integrated with bottom-up signals, such as saliency maps or movement detectors. The influence of different attention biases can be modulated according to the task, the perceived interaction stage, what we know regarding the partner, and so forth.

We consider top-down attention as a competition of resources between multiple inverse models that seek to confirm their hypotheses about what the demonstrator's action/intention is. The saliency of a request for resources from each inverse model can be linked to the quality of the predictions it offers. When applying these principles to a robot, the computational and sensorimotor resources of the robotic system are distributed to the different inverse models, as a function of the quality of the predictions they offer about forthcoming states of the interaction. Meanwhile, a continuous estimation of environmental affordances allows to dynamically update those inverse models which are applicable to the current state of the interaction. 
This way, the system is able to provide a prediction of the position of the observed agent effector, and thus an interpretation of the agent action. The system can also supply estimation of the location of objects in the environment, which constitute potential targets for the action being executed. Saccadic movements are then performed according to a certain confidence level attributed to each of the competing models, and to the saliency of a feature (either hand or object).

Previous approaches required knowledge of the features of the different targets present in the environment to detect them [5], or knowledge of their positions. In this work, we propose a model that can overcome these limits, allowing for simultaneous exploration of the environment and recognition of the actions, exploiting both sources of information to achieve faster action recognition. We further consider that visual information gets more reliable and less noisy moving from the periphery to the center of the visual field, following a foveal model of vision.

Experimental studies [29] have shown that during action perception in condition with low uncertainty on the action goal, humans consistently anticipate the movements and direct their gaze toward the targets. Our model shows similar anticipative behaviour and predicts the presence of an initial phase where attention is more explorative and less predictive when the uncertainty is higher.

The precise nature of the problem and related approaches are discussed in Sec. 2, the attention control system is introduced in Sec. 3, and its experimental validation described in Sec. 4.

\section{The problem}

Perception in active vision is constituted by a sequence of visual shots interleaved by saccadic movements[1, 2], aimed at purposefully exploring the environment, in order to extract information relevant for pursuing the current goals. Given this strategy, the quality of the obtained information is due in great part to efficient and intelligent gaze control. A fundamental issue on this regard is the implicit indetermination of attending to something we cannot precisely locate yet. This requires a concurrent evolution of both the knowledge regarding the environment and the quality of the attention strategy. In our case, to achieve a shared dynamical attention allocation during a social interaction, decisions on where to look are strictly linked to the movements of the partner. This adds further complexity to the task, which now has to account for a changing visual scenario. Human behavioural studies in visuo-motor control have shown that humans are able to adapt their visual exploration to the specific requirements of the task at hand $[17,11]$. The neural mechanisms underlying such top-down control of attention in visual exploration are only beginning to be unveiled [7]. Since visual exploratory behaviour is inherently costly, it is fundamental to optimise the relation between the value of the gathered information and the cost of obtaining it [23]. As described in detail below, we address this problem by trying to devise what is the best visual focus of attention at each moment during a social interaction. 
Robotic studies on adaptive active vision so far have focused on the previously mentioned topics in isolation. In [19] an artificial fovea is controlled by an adaptive neural controller. Without a teacher, this system learns trajectories causing the fovea to find targets in simple visual scenes and to track moving targets. The model in [10] solves active sensing problems under uncertainty. A reinforcement learning algorithm allows it to develop active sensing strategies to decide which uncertainties to reduce. However, in that study the model of the task is known a-priori and motor control is hardwired. Other works (e.g. [3, 21]) employ evolutionary learning techniques for developing adaptive active vision systems. These approaches are robust to the perceptual aliasing problem, however they do not allow on-line adaptation to changing environments. In [13] a neural architecture for eye arm coordination is proposed which learns autonomously taskspecific attentional policies, exploiting a strong link between attention and execution of actions. The authors also proposed that a bottom-up attention system can be exploited to bootstrap learning, and hypothesised on the basis of neural simulations that the limited size of fovea can play an important role in the efficiency of learning [15]. [32] proofs the theoretical advantages of using an active gaze strategy for efficient object class learning.

In the present work, we deal with the above issues by letting an integrated attention system assume gaze control while observing a partner performing a reaching action toward one of a small set of target objects. Neither the goal of the action, nor the exact location of the potential targets are known beforehand. Consequently, the hand trajectory and target position have to be estimated contextually while trying to understand what is the action goal, i.e. where the partner is moving its hand towards. An example of a possible experimental setup is provided in figure 2, where the humanoid robot iCub is observing a human partner starting a reaching movement towards one of three potential target objects placed in the common working space. The robot has to decide where to observe (estimated hand or object position) in order to a) estimate the objects exact positions and b) understand where the partner's hand is reaching at.

\section{Action recognition with dynamic allocation of attention}

We build on some of the concepts introduced with the HAMMER (Hierarchical Multiple Models for Execution and Recognition) architecture for action perception and imitation based on 'direct matching hypothesis' [4]. The system described here is based on the integration of the latest HAMMER framework implementation [18] with a gaze controller which directs attention in order to maximise discrimination performance, while maintaining robustness to noise, and a contextual estimation of both end effector location and position of all potential targets.

A number of different models, at least one for each of the possible targets of the action, compete for both attention allocation and for the final discrimination of the action goal. Following HAMMER guidelines [5], the discrimination between the available action hypotheses is based on the computation of a confidence value that measures the 

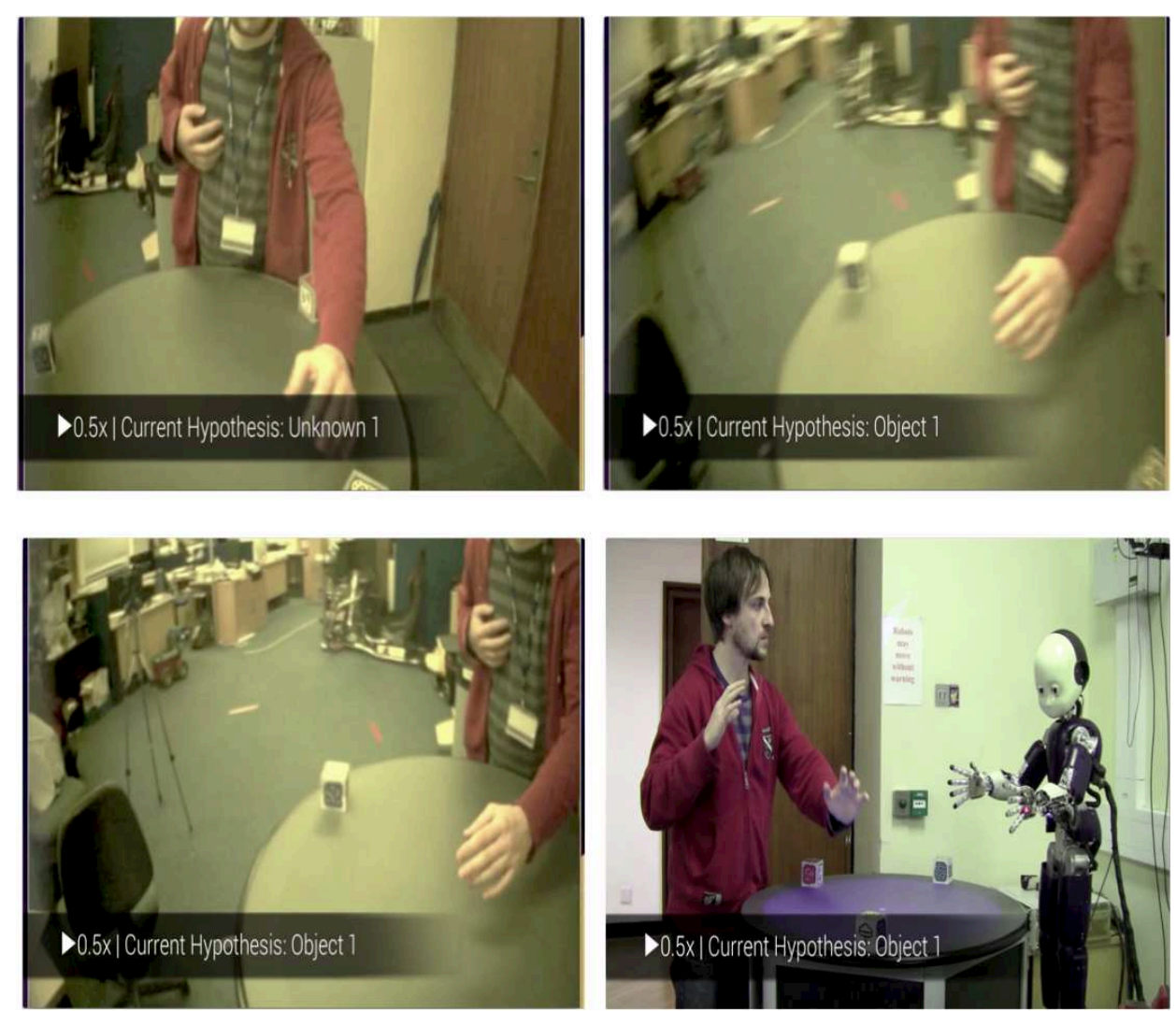

Figure 2. Example of experimental setup, with iCub looking at target objects and arm movements (bottom right). In the top-left the iCub's gaze is following the hand, than, top-right, the gaze anticipate the hand movement, finally, bottom-left, the gaze is focused on the target object before the hand reaches it. These three images are grabbed from the iCub camera during an interaction trial.

overall Euclidean distance between the predicted action trajectories and the observed motion trajectories. To compute such prediction, HAMMER uses a combination of forward and inverse model pairs which are the same models used for action control.

Formally, an inverse model is a function that, given a certain goal $g$, maps the current state $S$ to the action $A$ which the agent has to execute to achieve the goal: $i_{g}: S \rightarrow A$. A forward model is a function that maps the current state and the action being executed to the next expected state $f_{g}: S \times A \rightarrow S$.

In this work, the candidate actions among which the observer has to chose are different reaching movements toward different targets in space, $g$. A reaching model $m_{g}$, composed of a pair of inverse and forward models $\left(i_{g}, f_{g}\right)$ is required for each different $g$. Each reaching model works directly in the space of the end-effector, and the action space is coincident with the state space $(A \equiv S)$, because the used inverse model computes the next desired end-effector position $\mathbf{p}^{t+1}$, and the forward model returns the same value.

The following is the equation, akin to a PD controller, employed by a model $m_{g}$ to compute the next position $\mathbf{p}^{t+1}$, when the target is at position $\mathbf{p}_{g}$ :

$$
\mathbf{p}^{t+1}=\mathbf{p}^{t}+\tau\left\{\dot{\mathbf{p}}^{t}+\tau\left[K\left(\mathbf{p}_{g}-\mathbf{p}^{t}\right)-D \dot{\mathbf{p}}^{t}\right]\right\} .
$$


This equation leads to a motion with a smooth linear trajectory that asymptotically reaches the target. The confidence function for each model and time step $c_{t}^{g}$ is updated employing the difference between the predicted end-effector position $\tilde{\mathbf{p}}^{t+1}$ and the perceived one $\mathbf{p}^{t+1}$ :

$$
c_{t+1}^{g}=\frac{1}{1.0+\left\|\tilde{\mathbf{p}}^{t+1}-\mathbf{p}^{t+1}\right\|}+c_{t}^{g} .
$$

The above confidence equation is unitless, and valid only for the qualitative comparison among different models. Whilst it is used in the example tests described at the beginning of the next section, in the rest of experiments the analysis is based on the ratio of trials with correct action recognition. To produce a response to a perceived action, it is necessary to take into account that the confidence values depend on the duration of the action and the initial distance between the effector and the target.

For increased plausibility, we assume that the observations of the end-effector and of the affordances are affected by noise that is dependent on the sensors configuration, i.e. gaze position. Thus, if the observer gaze position is $\mathbf{p o s}_{o}$, the actual observation of an object at position $\mathbf{p}$ is distributed according to:

$$
\mathbf{N}\left(\mathbf{p},\left(\sigma_{f}+\sigma_{d}\left\|\mathbf{p}-\mathbf{p o s}_{o}\right\|^{2}\right) \mathbf{I}\right) \text {. }
$$

With $\sigma_{f}=0.1$ and $\sigma_{d}=0.2 \ddagger$. This noise model represents an approximation of human foveal vision, where most of the visual receptors are located in the central area of the retina (fovea) and their density - and the visual resolution - decreases departing from the fovea.

In order to deal with noisy inputs, each model uses Kalman filters for the estimation of the end-effector and affordance positions, and an active vision system is integrated that exploits the estimation of the uncertainty produced by the Kalman filters. The main assumption is that the observer can use features which allow to discriminate between the different affordances and the effector. This approach is similar to that of [9] and [20] but, instead of being limited to track or to find objects in a dynamic environment, it allows for active recognition of a dynamic event.

In this work, independent Kalman filters are used for each action element. An action element has position $\mathbf{p}$ and produces an observation $z$. The associated Kalman filter produces an estimated probability distribution $b_{\mathbf{p}}$ and a corrected probability $\hat{b}_{\mathbf{p}}$, which uses the observation received at the current time step.

In order to produce these estimations the Kalman filter uses a process model and noise model. The process model has the form $\mathbf{p}(\mathbf{t}+\mathbf{1})=\mathbf{A p}(t)+\mathbf{b}(t)$. For both the effector and the targets, matrix $\mathbf{A}$ is the identity matrix $\mathbf{I}$. We assume that the targets are still, thus their process noise $\mathbf{b}(t)$ is zero. The effector process noise is $\mathbf{b}(t)=0.01 \times \mathbf{I}$ to model small changes in the trajectories.

We decoupled the prediction and the update phases of the Kalman filters for the end-effector. In each model, after the update of the estimated position of the target

$\ddagger$ The effect of these parameters on the system’s performance will be studied in section 4.3 and 4.4. 
and the prediction of the end-effector position (so without before taking into account the observation) is updated in accordance with the action model in eq. 1. This step modifies only the estimated position while leaves the variance untouched. After this step the Kalman correction phase for the end-effector is executed.

In our task, we need to take into account the implicit imprecision of the sensory information, together with the lack of exact knowledge regarding the position of the targets and the end effector. As a consequence, the typical Kalman formulation has to be adapted, so that the observation models of the Kalman filters are able to account for the change in sensory configuration and uncertainty regarding the real environment. While observation noise increases with the distance between the observation point and the real object position, the latter is not known; only a prior estimation $b_{\mathbf{p}}=\mathbf{N}\left(\overline{\mathbf{p}}, \boldsymbol{\Sigma}_{p}\right)$ is available before saccade execution. Thus, the resulting observation model depends on current gaze position and belief state:

$$
P\left(\mathbf{z} \mid \mathbf{p o s}_{o}, b_{\mathbf{p}}\right)=\int p\left(\mathbf{z} \mid \mathbf{p}, \mathbf{p o s}_{o}\right) b_{\mathbf{p}} d \mathbf{p} .
$$

The implemented observation model is expressed by the following normal distribution, considering that Kalman filters assume Gaussian distributions and linear dynamics§:

$$
\left.P\left(\mathbf{z} \mid \mathbf{p o s}_{o}, b_{\mathbf{p}}\right) \approx \mathbf{N}\left(\overline{\mathbf{p}},\left(\sigma_{f}+\sigma_{d}\left\|\overline{\mathbf{p}}-\mathbf{p o s}_{o}\right\|^{2}\right) \mathbf{I}+0.7 \sigma_{d} \boldsymbol{\Sigma}_{p}\right)\right) .
$$

The attention system uses the probability distribution estimated by the Kalman filters in all the models and the confidence value of each action hypothesis. The attention system operates to minimise the uncertainty of the elements in the most probable action hypothesis. The effects of reducing uncertainty between the different action hypotheses are not directly taken into account by the current system for computational reasons. Gaze point selection currently considers only instantaneous saccades even if the system is allowed to select a new saccade target only after the previous attentive action has been completed.

Each element of each model, e.g. target and effector, is considered independently by the attention system instead of integrating the different probability distributions associated to elements shared by different models. e.g. the different expected positions of the end-effector for the different models. In our implementation, each action hypothesis has two elements: effector and affordance; and the attention system selects targets from a set of $2 * n_{a}$ elements, where $n_{a}$ is the set of action hypotheses.

The selected target, with estimated position $\overline{\mathbf{p}}$ related to hypothesis $g$, is the one which maximizes the following objective function:

$$
\ln \left((2 * P I * e)^{N}\left|\boldsymbol{\Sigma}_{p}\right|\right)\left(1+c^{g}\right) .
$$

$\S$ The observation model differs from the actual observation generation model of eq. 3 because of the term $0.7 \sigma_{d} \boldsymbol{\Sigma}_{p}$. This was added to take into account state and noise correlation which is usually not considered in Kalman Filtering. 


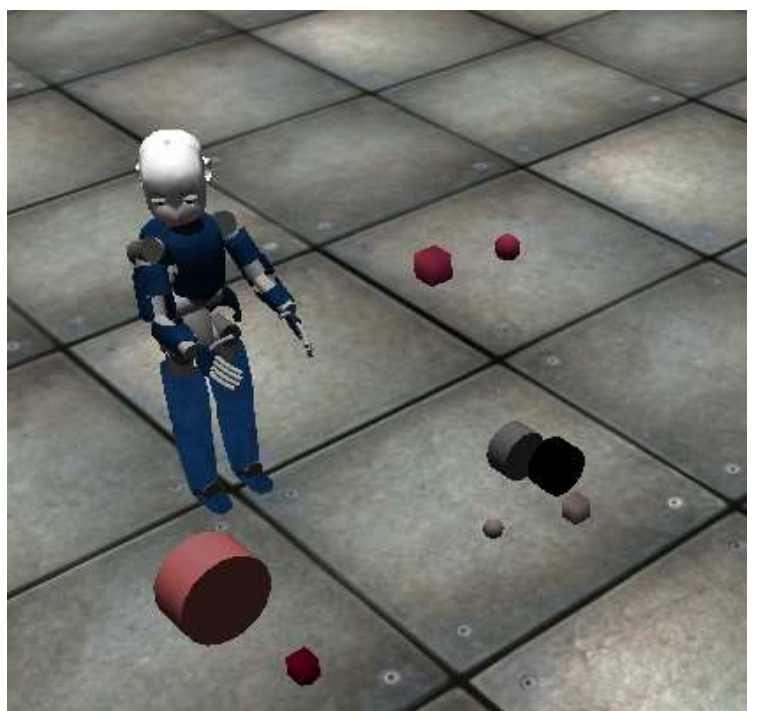

(a) Fixating laterally

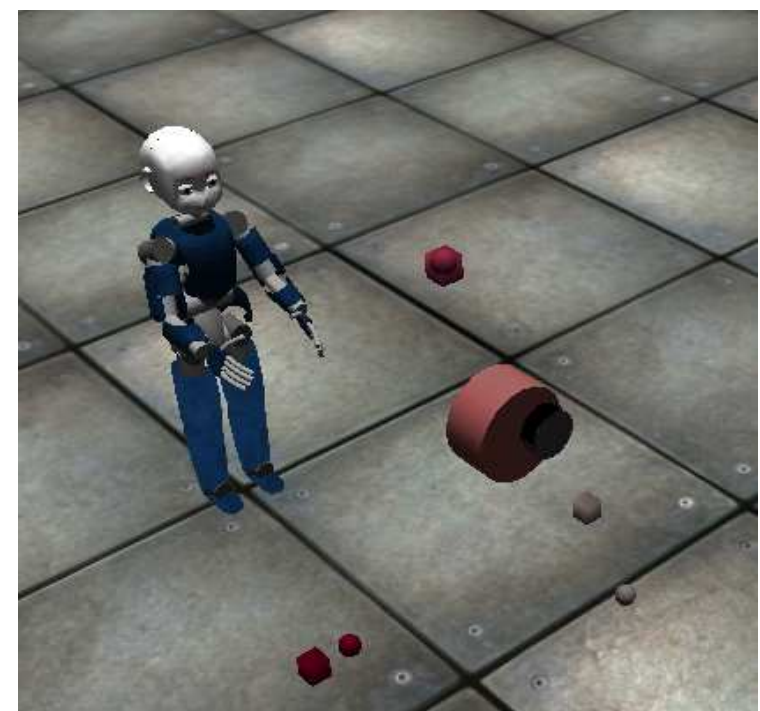

(b) Fixating at the hand

Figure 3. Experimental setup with example of different gaze points. The big red cylinder represents the gaze point of the robot. The boxes represent the real position of the observed action target while the small spheres represent the related observations that the robot senses. The dark small cylinder represents the end effector that is executing the action and the brighter one the related observation.

This objective function accounts at the same time for both the reduction of uncertainty, which can be measured using entropy, and the relevance for the most likely action hypothesis.

There are several approximations in this objective function: a) it does not consider the residual entropy of the target assuming that after the saccade the object will be perfectly centered; b) it does not consider the information gain on the other targets. At the same time, this formulation allows to select only positions corresponding to estimated targets, while other positions may allow to increase the overall information gain.

\section{Experimental evaluation}

The proposed model has been implemented on the iCub Simulator [31] where the simulated robot head was controlled by our attentional system. In this section, after describing the experimental setup, we present and discuss the results we have obtained with different parameters and in various working conditions. 


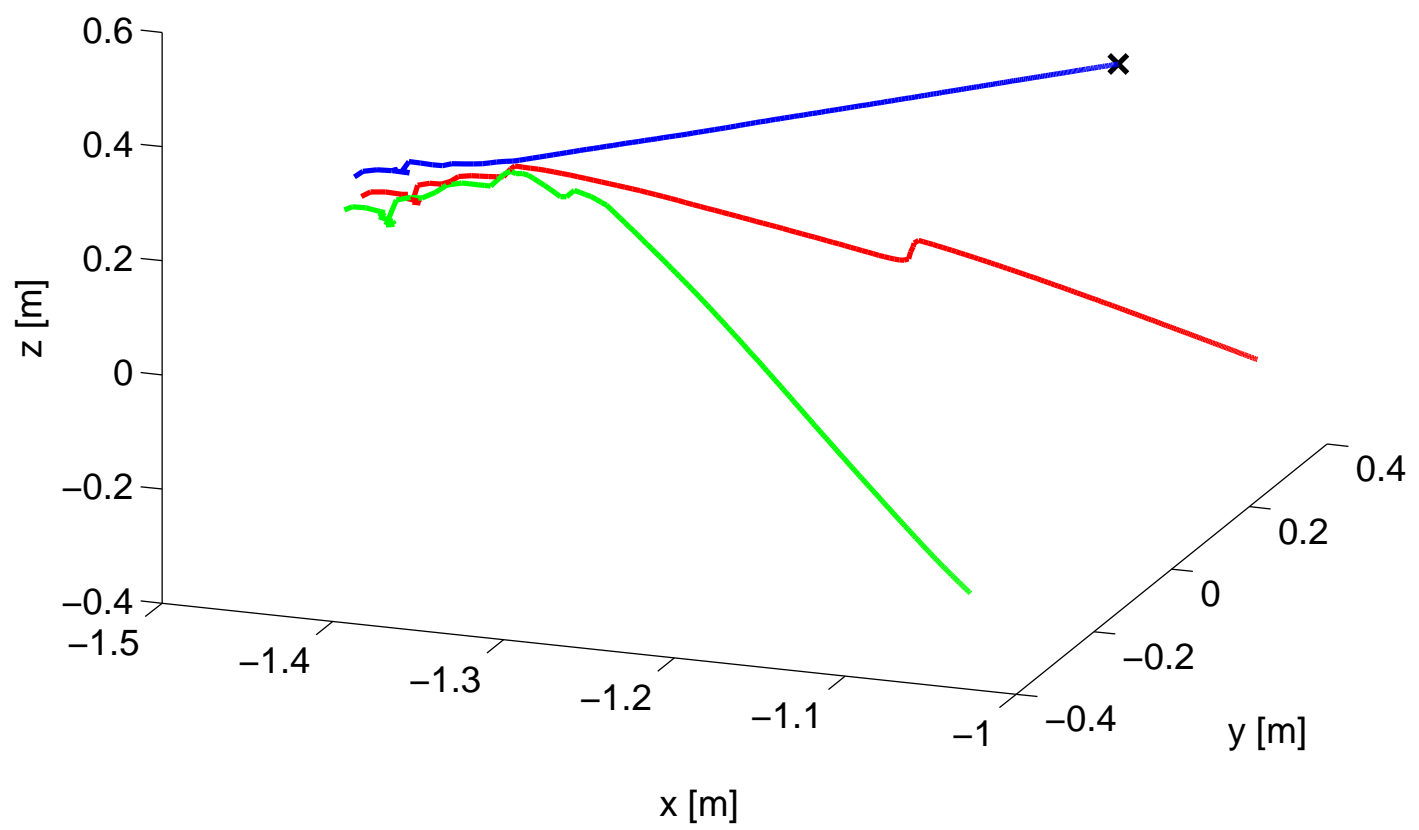

Figure 4. Estimated hand trajectories according to the three competing models, which converge asymptotically to the three targets. Sudden changes in the two lower estimated trajectories are due to gazing actions that change the related observation models.

\subsection{Setup}

First of all, a variable number of target objects are created in the simulated environment (see the three coloured boxes in figure 3)\|. The system receives noisy observations, represented as small spheres of the same colour as the actual objects. The simulated sensor noise is proportional to the square of the distance between the real position (boxes) and the robot gaze point (red cylinder), following Eq. 3. The end-effector of the other agent is displayed by a black cylinder, while its observation is a grey cylinder. The head and eye are controlled using the fixation point and the iKinGazeCtrl iCub module [30]. A new fixation point is sent only when the previous movement has ended.

The end-effector of the other agent is moving, according to equation 1 with $K=3$ $D=0.5$ and $\tau=0.04$, towards one of the target object, randomly selected. The system is provided with a set of models, one for each target object, with prior $\mathbf{N}(o, I)$, where $o$ is an observation sampled according to the noise model, constituting the initial gaze point. Examples of hand trajectories devised by the concurrent models are depicted in figure 4. It can be observed how the hand terminates in each of the three target objects corresponding to the three candidate models. The top trajectory is the one which is actually performed in this case.

A typical evolution of the confidence level for each of the three competing models can be observed in figure 5 for both Moving gaze and steady gaze protocols. It is

|| The system supports online detection of new objects. For every object detected, new models are instantiated whose initial confidence is equal to the average confidence of the already existing models. However, the performance of the system when such events occur is not examined in this paper. 
again possible to observe how the active paradigm is able to differentiate the goal action around time step 20 (figure 5(a)), whilst the passive paradigm seems to fail completely in the task (only at the very end of the trial a small, still non-significant prevalence of one of the models can be spotted, figure 5(b)).

To better understand how the active exploration of the environment through the execution of saccadic movements is performed, figure 6 shows an example of the evolution of gaze direction during an experiment, computed by the attention model as described in the previous section. Three different phases are highlighted. During the first half of the trial (dots in figure 6), gaze moves rather erratically all around the task space, but after this bootstrapping phase more regular behaviours can be observed. Time steps from half to three quarters of action execution show gaze points approximately distributed along the dominant hand trajectory, suggesting that the system has now understood where the action is going on (plus symbols in figure 6). Finally, in the last quarter of the trial, one of the model seems to be clearly dominant over the others. Estimations of both hand trajectory and location of target object are reasonably accurate, and the dominant model makes the system move forth and back between these two locations, which are now definitely considered the most interesting, to further improve their estimation (circles in figure 6) 9 .

The last type of analysis we present concerns the actual prediction capabilities of the system in terms of estimation of hand trajectory and object position. figure 7 shows the error observed in the approximation obtained by each model in its estimation of the effector trajectory and target location. It can be observed that only one of the models achieves a correct estimation of the actual hand position (i.e. the model that

व Note that the oscillation between the effector and the target is due to an increase of the uncertainty of the unobserved object.

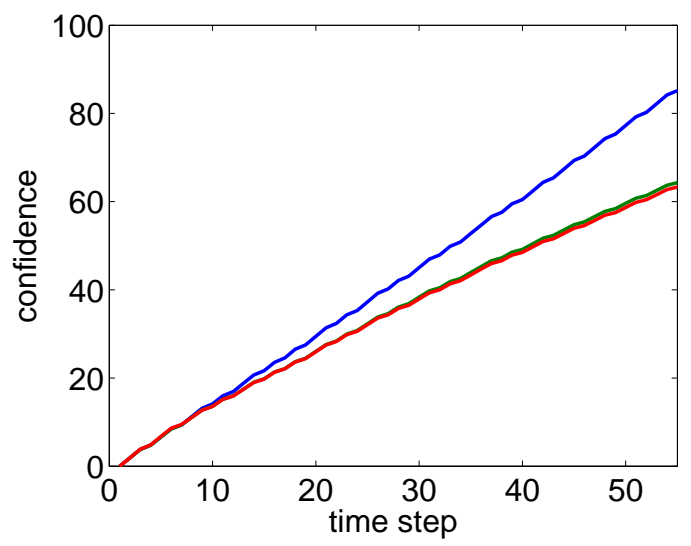

(a) Moving gaze

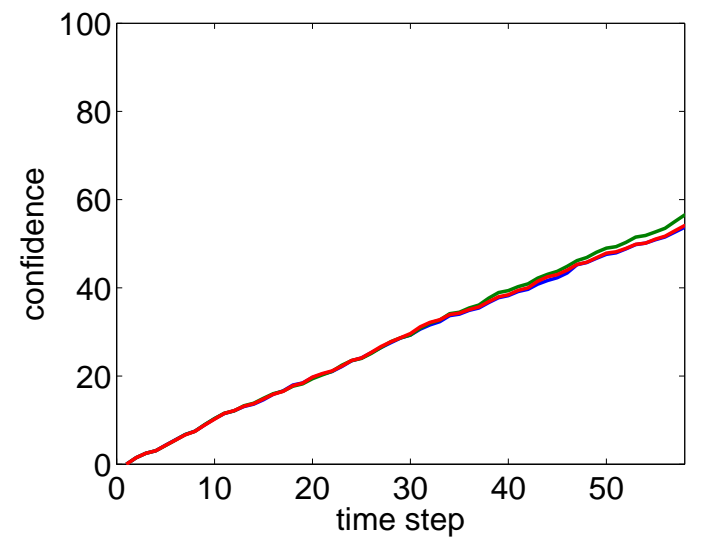

(b) Steady gaze

Figure 5. Evolution in time of confidence with (a) and without (b) attentional gaze control. With gaze control the right action is clearly identified before time-step 20 . Without gaze control the agent is not able to recognize the performed action. 


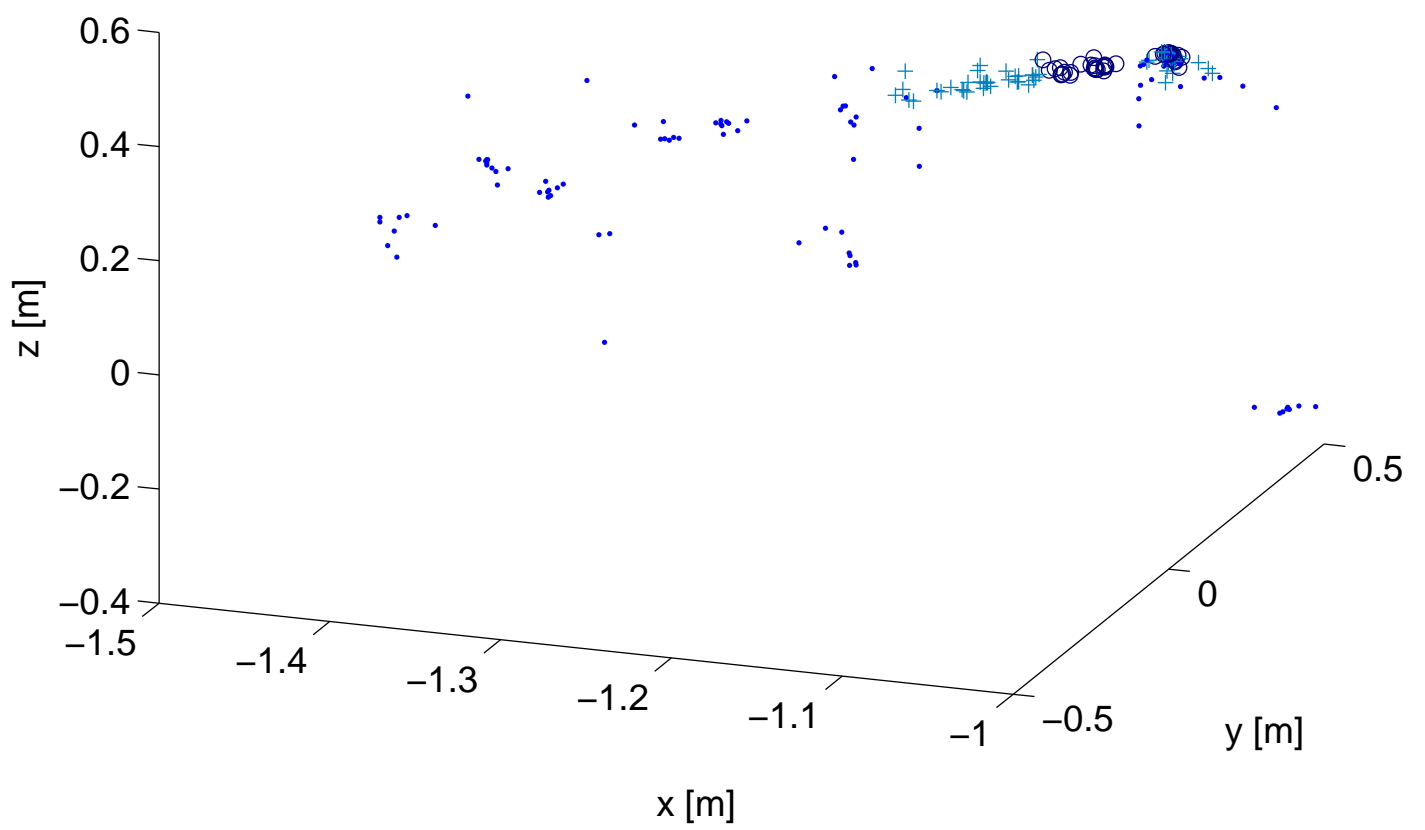

Figure 6. Evolution of gaze point during different stages of action observation (dots: first half; plus: third quarter; circles: fourth quarter).

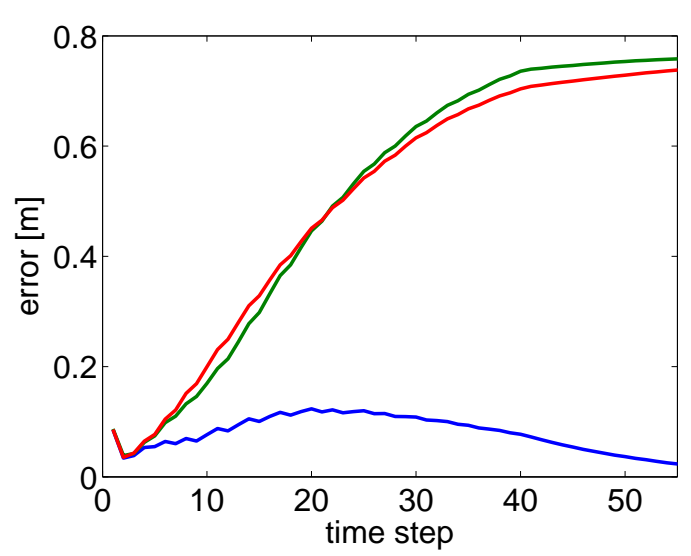

(a) Hand estimation error

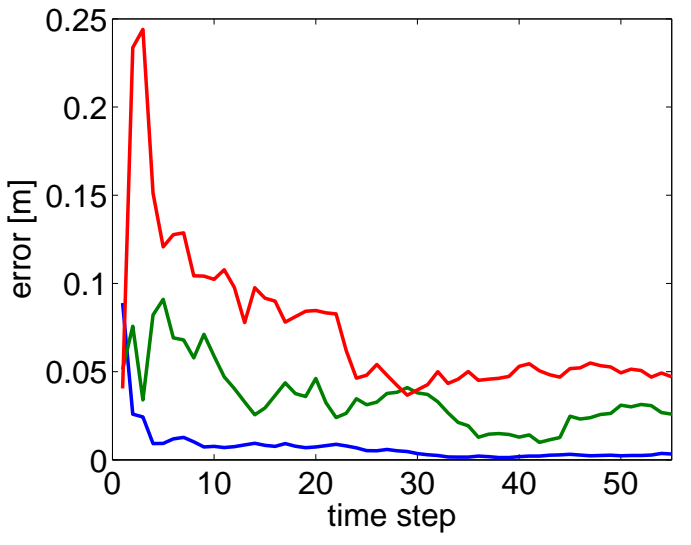

(b) Target estimation error

Figure 7. Evolution of estimation error on hand position (a) and on target position (b) according to the three competing models. End-effector position is estimated correctly only by one of the models (a), while target location is estimated with good approximation by each model, reaching a $50 \mathrm{~mm}$ error in the worst case.

correctly recognises the action), whilst the others wrongly converge towards the wrong targets (figure 7(a)). Nevertheless, figure 7(b) shows that even the targets associated to the losing models are detected with a good approximation, and the first of the phases depicted in figure 6 is critical in this regard, as it allows to achieve a good representation of all stimuli in the environment while gradually shifting the focus toward the supposedly most interesting one.

Next, we describe the predictive capabilities of the system comparing its 
performance in different environmental conditions and with different attentional strategies.

\subsection{Results}

To study in detail the role of active gaze control in visual action perception we compared different configurations of the action recognition model in different environmental configurations. We considered three different visual attention control strategies:

Ideal VAC (Visual Attention Control) this strategy uses the proposed attention control law to select the gaze targets; the related robot head-eye controller performs ideal, instantaneous movements to gaze the targets, and is implemented by stopping the evolution of the environment while the robot head is moving.

Real VAC this strategy uses the same attention control law as the previous one, but the head-eye controller of the robot is subject to motor delays and noise in the motion trajectory, simulating a realistic real world implementation [30].

No VAC this strategy does not employ any gaze control target during action perception.

In each configuration of the environment, the target objects were disposed on a circle placed on a vertical plane in front of the robot. All objects were always at the same distance from their centre of symmetry located in front of the performer, and remained visible by the observer at all times. The initial performer position was at $0.4 \mathrm{~m}$ from the centre of the circle of objects. The initial gaze point was selected in a stochastic manner at the start of each trial to be near $(d<0.2 m)$ one of the targets or the performer effector. This should allow a fair comparison between the models. We modulated two parameters of the environment, making them assume three values each, for a total of nine conditions:

(i) The number of objects (2, 4 and 6);

(ii) The object distance from their centre of symmetry (close $=0.2 \mathrm{~m}, \mathbf{m e d i u m}=0.4 \mathrm{~m}$ and $\mathbf{f a r}=0.8 \mathrm{~m}$ ).

The head-eye controller was set with a trajectory execution time of $0.2 \mathrm{~s}$ when the angular distance between the initial and final gaze positions were less than $6 \mathrm{deg}$, and $0.5 \mathrm{~s}$ otherwise. In the Real VAC model the final gaze target was sent to the head-eye controller at each time step, without waiting for the controller to reach its final position. The average trial duration is $7 \mathrm{~s}, 8.4 \mathrm{~s}$ and $10.3 \mathrm{~s}$ for the different distances, corresponding to 13,15 and 18 timesteps on average. The element positions perceived by the robot were affected by gaussian noise with standard deviation $\sigma=\sigma_{f}+\sigma_{d}|\mathrm{X}-\theta|$, with $\mathrm{X}$ the position of the element and $\theta$ the current gaze focus position, $\sigma_{f}=0.1$ and $\sigma_{d}=0.2$. We must note that for the close distance condition the fixed standard deviation $\sigma_{f}$ has a value which is just half of the distance between the objects. This means that, independently from the attentional behaviour, the distance between the trajectories to different targets will be small compared to the perceptual noise. 


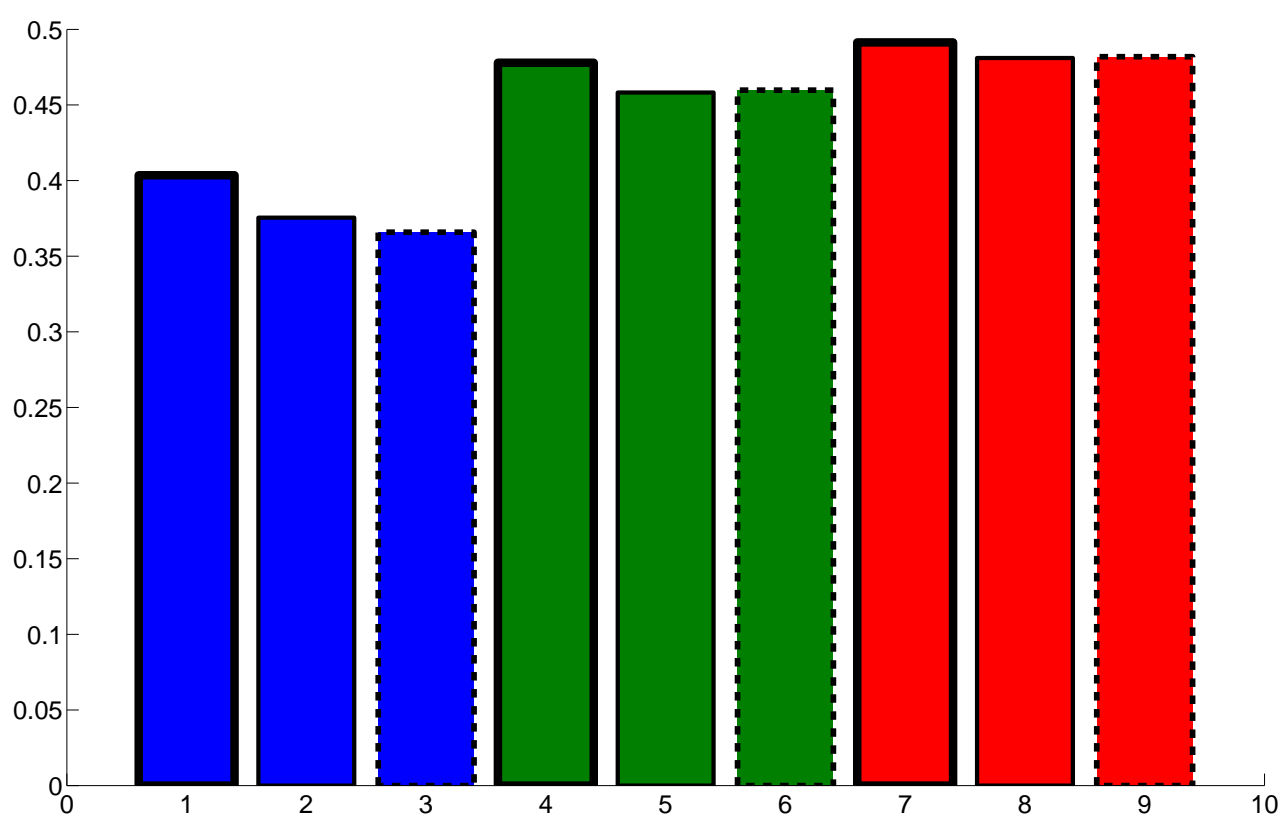

Figure 8. Predictive recognition index in environments with 2 objects. Color coding refers to the distance of the target objects: blue $=$ close (bars 1, 2, 3); green $=$ medium (bars 4, 5,6); red = far (bars 7, 8, 9). Bar border style refers to the attention strategy used: thick line $=$ ideal attention (bars $1,4,7$ ); thin line $=$ realistic attention (bars 2 , $5,8)$; dashed line $=$ no attention $($ bars $3,6,9)$.

For each of the 9 conditions 1000 trials were executed.

In order to compare the behavioural usefulness of the different strategies and their capability of providing early responses, we introduced the predictive recognition index $c$ :

$$
c=\frac{\sum_{t=1}^{N} t f(t)}{N(N+1)}
$$

where $f(t)$ is the ratio of trials with correct action recognition at timestep $t^{+}$. Using this index we can evaluate not only the final recognition rate but also its progress. This index will increase when the observer is faster in recognising the correct action goal. In the integration it still associates higher weight to later estimations, when the observer has accumulated more information. This is important because a response controller can actually decide when to answer on the basis of the expected increase in the estimation quality.

Figures 8, 9 and 10 display the value of $c$ respectively for 2, 4 and 6 objects. As a general consideration, the overall performance indicates that task difficulty increases with the number of objects, and decreases with the distance between the objects.

+ Action length was normalised to the average length of the actions for each distance and number-ofobjects condition. 


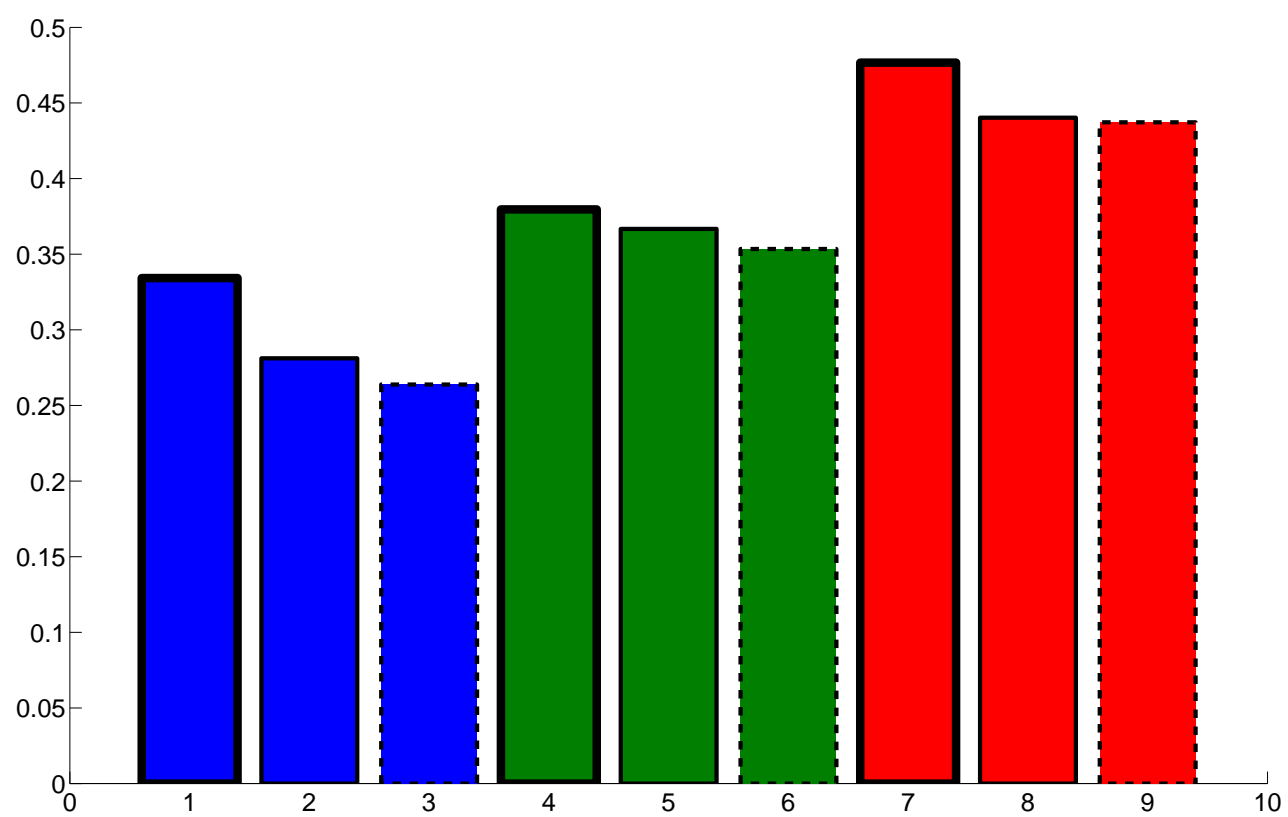

Figure 9. Predictive recognition index in environments with 4 objects. See 8 for a description of the figure features.

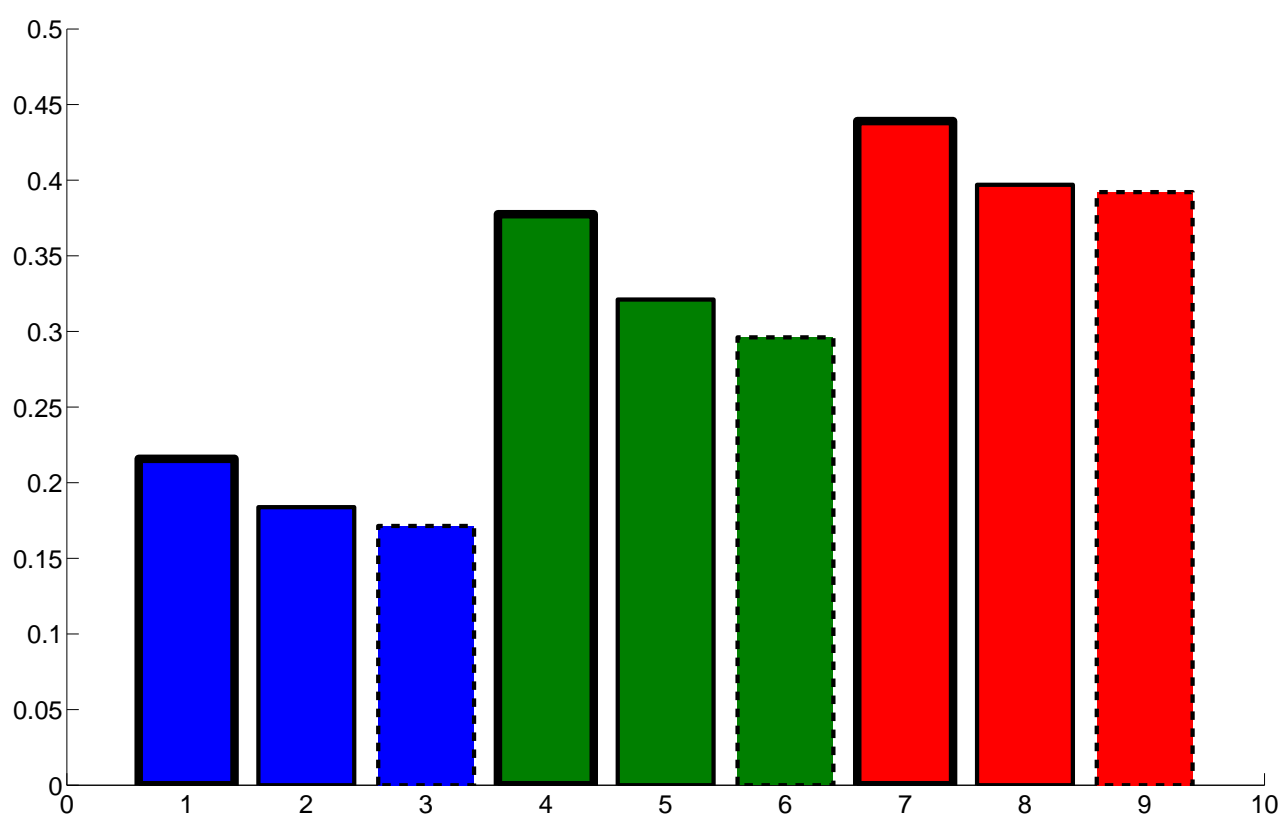

Figure 10. Predictive recognition index in environments with 6 objects. See 8 for a description of the figure features. 
Comparing the control strategies once fixed the environmental conditions, the Ideal VAC strategy clearly outperforms the Real VAC and the No VAC. On the other hand, it appears that the proposed approach is sufficiently robust to be used with a real robot in a dynamic environment, as the Real VAC strategy provides consistently better results than the No VAC in all conditions, though the advantage is reduced for larger distances. In general, the advantage of active gaze control is higher when the task is more difficult, i.e. when there are many near objects (with 6 objects, close, the improvement is 26\%) than with fewer objects far away (with 2 objects, far, the improvment is only $2 \%$ ). This is likely due to the increased noise on the gaze position control when the distances between two successive gaze targets increases.

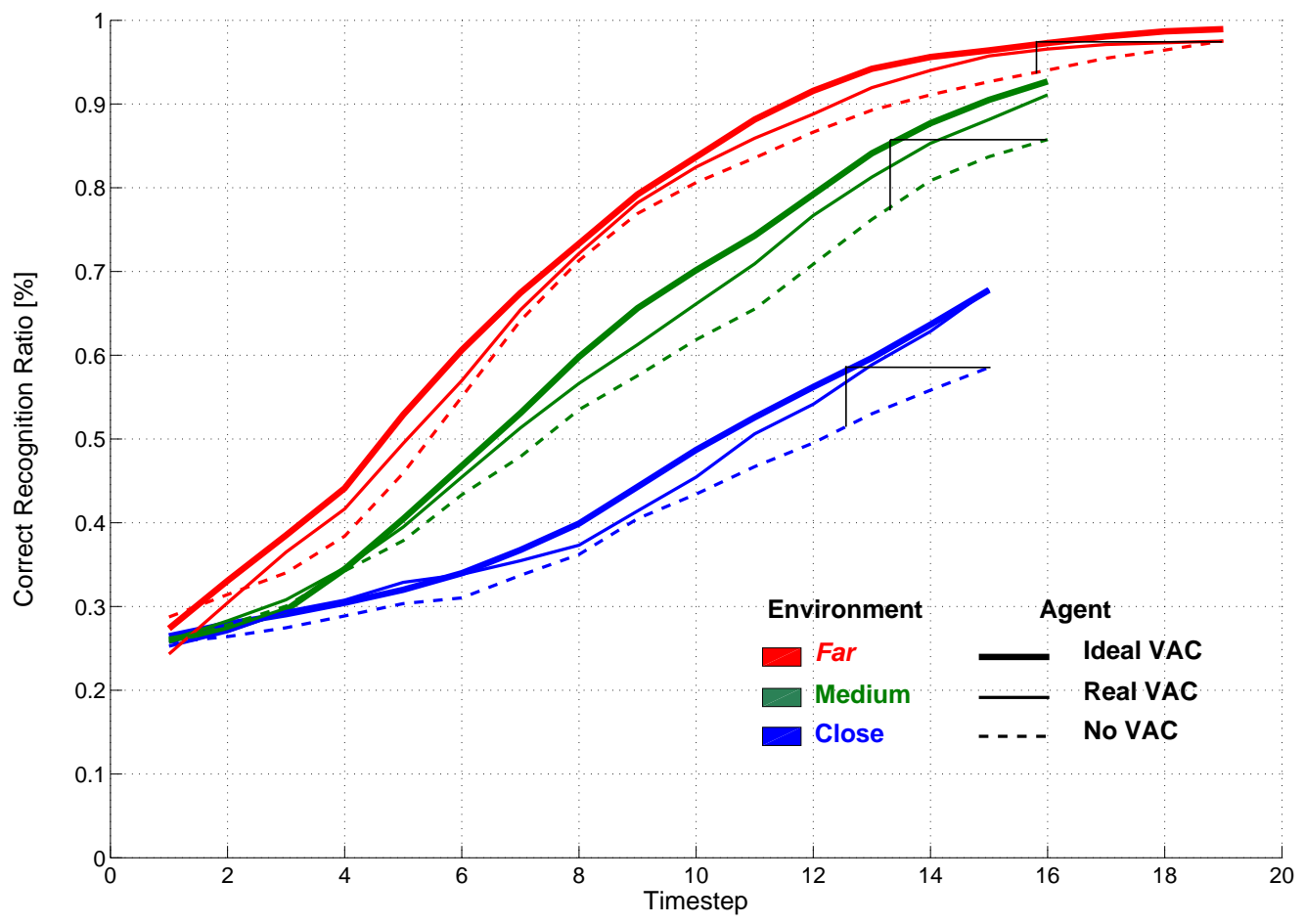

Figure 11. Evolution of recognition ratio during a trial with 4 objects (average). The black lines show the temporal advantage of the Ideal VAC over the No VAC model with the same recognition ratio.

Figure 11 displays the evolution of the average correct action recognition during the trial. It clearly shows that the active gaze control delivers an advantage in the hard close condition, we can also notice that in the simpler medium and far conditions the models with an active gaze control present a faster increase of the recognition index. As it will be shown (see figures 12 and 13), this is due to a faster reduction in the position estimation error from the initial estimation allowed by active sensors control. In each condition, the active controllers consistently surpassed the performance of the passive system. Furthermore the Ideal VAC controller reached the passive controller 
maximum recognition rate in approximately $20 \%$ less time. The Real VAC also shows a significative, but slightly smaller, improvement. This allows a considerable temporal advantage to anticipate the preparation and even the execution of any response action [27]. Finally, figure 11 confirms that the Real VAC model is slightly less favoured in the far condition in terms of initial recognition rate, while in the other two conditions its initial performance are almost good as those of the Ideal VAC. This is due to high initial uncertainty on the object positions due to their reciprocal higher distance and thus the distance from the initial gazing target which is randomly selected near to one of the targets. The active initial exploration is important to reduce this high initial uncertainty but it may involve several long distance gaze movements which are slower and less robust with the Real VAC model.

We observed that the distance between the targets in the close condition is of the same dimension of the fixed observation standard deviation $\sigma_{f}=0.1$. This factor, together with the smaller number of observations collected during the shorter trajectories caused by nearer targets, explains the limited recognition ratio achieved by the system.

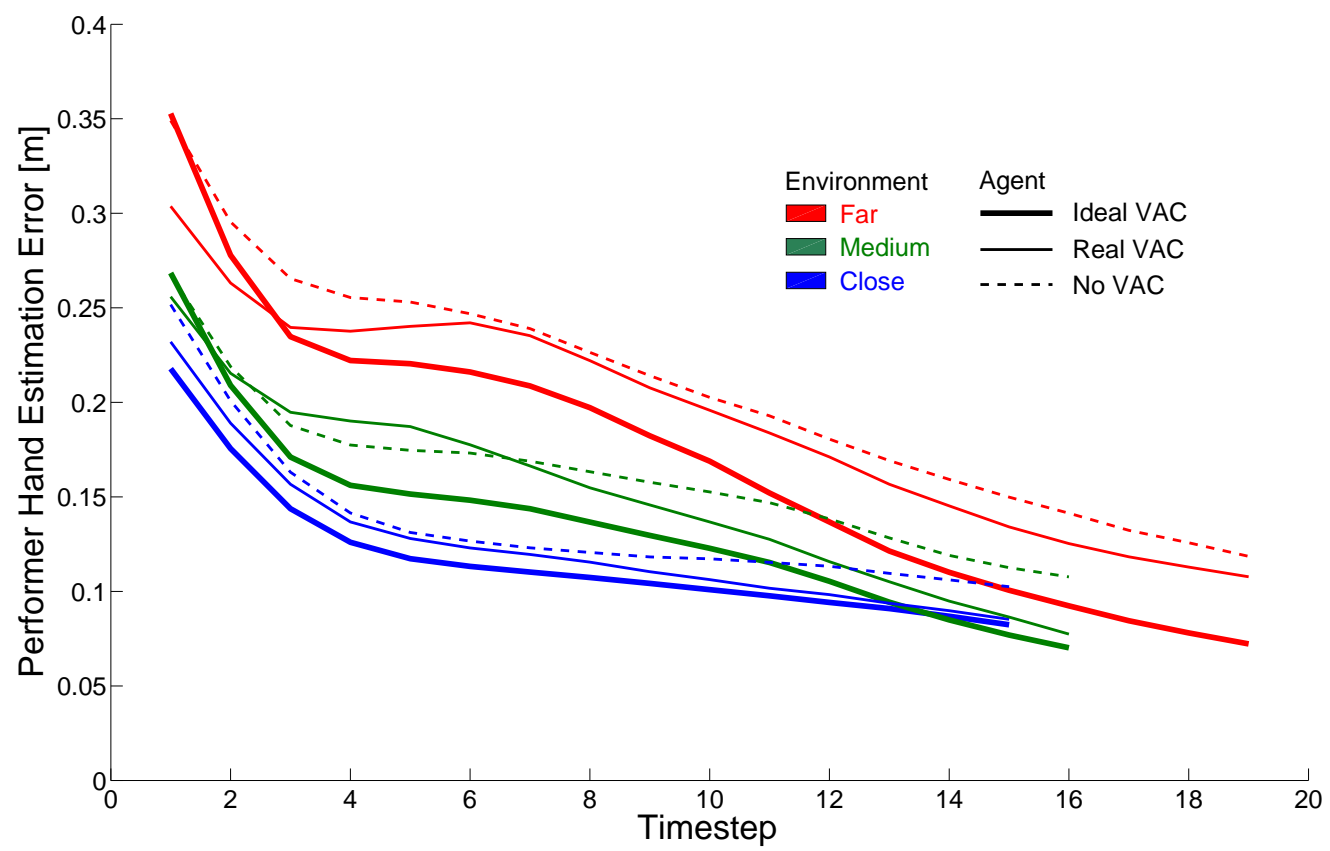

Figure 12. Evolution of hand position estimation error during a trial with 4 objects (average).

Figures 12 and 13 display the evolution of the estimation error on the state of scene elements (position of the effector and of the targets respectively) in the different conditions. They show again that the active gaze control delivers a substantial advantage in terms of precision and speed of convergence. The latter explains the performance improvement described above. An interesting behaviour shown by these figures is that in the far and medium conditions the estimation error seems to reach a stable condition 


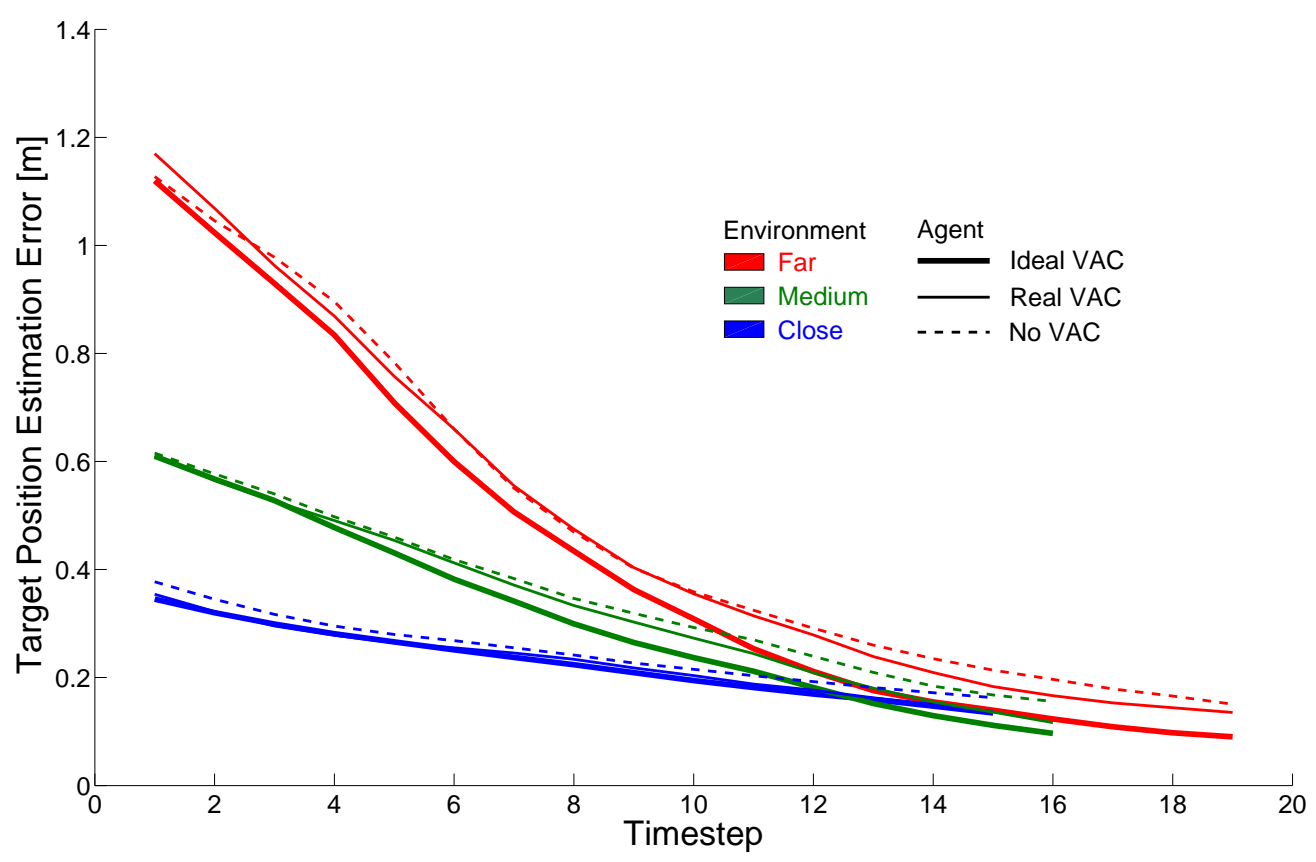

Figure 13. Evolution of target position estimation error during a trial with 4 objects (average).

before continuing its decrease, while the estimation error of the target decreases in a more continuous fashion. This can be explained by a gaze allocation behaviour focussed on the targets, as shown in figure 14, and by a strong noise of peripheral observations with this higher distances.

Figure 14 illustrates the evolution of the average gaze allocation during action execution in terms of the ratio between the gazing directed toward the target and toward the performer predicted by the model with highest confidence.

In far and medium conditions there is initially an approximately equal allocation to the predicted locations of the targets and the effector, then in all the conditions a higher priority is given to the targets while still allocating roughly $30 \%$ of the gazing actions to the target. One of the reasons for this behaviour is that the initial condition for the effector position is the same for all the action hypotheses. Therefore, during the initial phase, a gaze action directed to the effector position predicted by any of the action hypotheses reduces the uncertainty on the effector position estimated for all the hypotheses (see eq. 3 and eq. 5 for the relation between noise and distance).

The distance between the targets does not allow to get accurate observations of one element when observing another element (in far and medium conditions). During the initial phase, the system has high uncertainty on the actual event (and thus a low recognition ratio, see figure 11) thus the observations to the estimated target position are often not useful to estimate the position of the correct target which will be discovered later and will have accumulated a relatively high uncertainty level, particularly in the 
far condition.

In the close condition, the small distance between the elements permits an effective use of the peripheral vision, which, in this condition, allows the acquisition of relatively reliable observations for all the elements at the same time. This, later, translates in an increase of the saccades to the predicted position of the effector.

In the medium condition the system shows a different behaviour. It rises the allocation of saccades to the targets and successively reduces it. In this condition the uncertainty on the target is explicitly reduced by the gazing actions and then the system alternates between target and effector. The far condition does not present a reduction of the saccades to the target because the high distances does not allow a fast reduction of the uncertainty. However the same high distances produce big difference in the predictions of the various models allowing a fast event recognition, as shown in figure 11 (in contrast to a slower position estimation, as shown by figures 12 and 13).

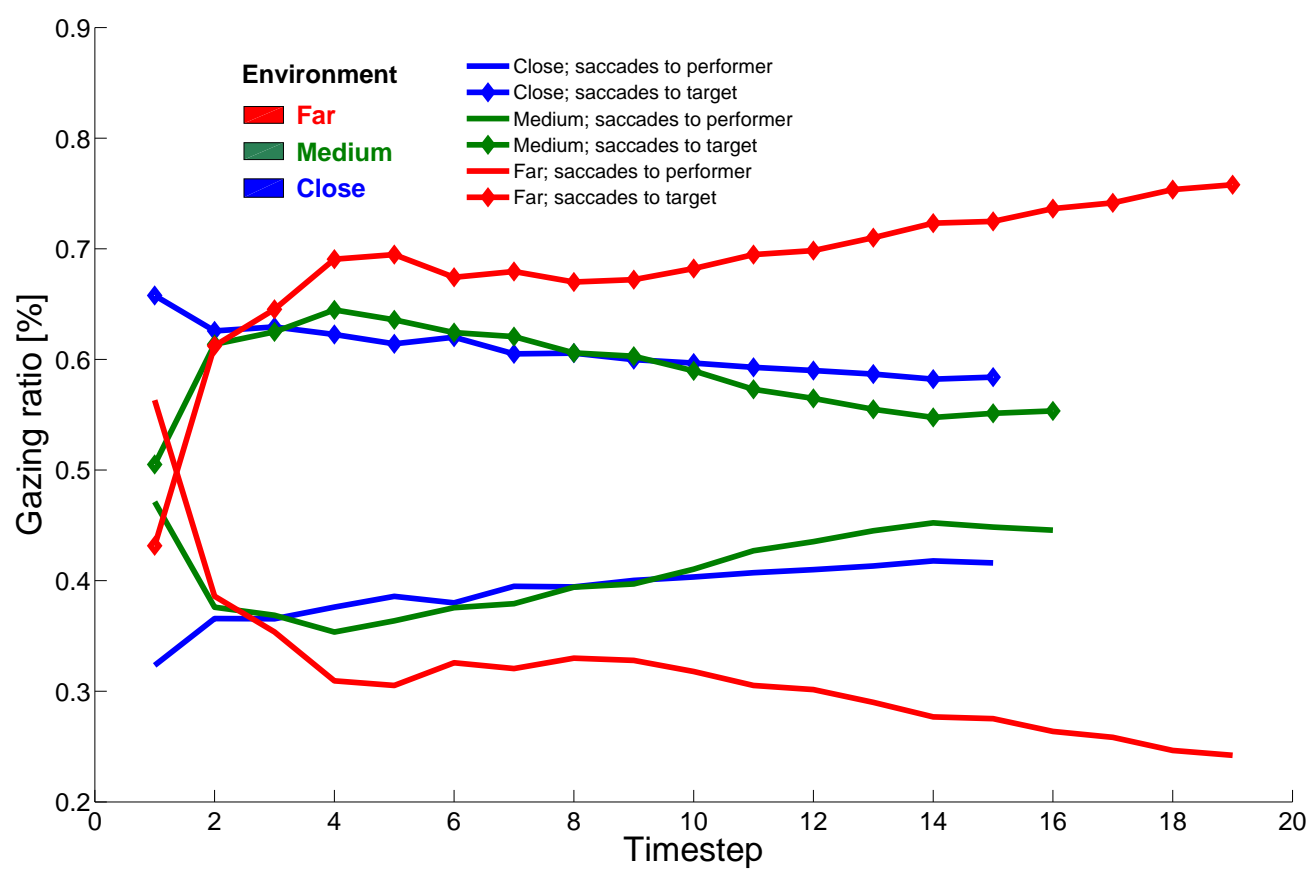

Figure 14. Ratio of gazes toward the predicted position of the performer's hand and the target object for the action hypotheses with highest confidence of the Ideal VAC for three distances. The sum of hand and object gaze time is not exactly 1 , as few saccades are executed to feed the other action hypotheses. Those are not shown for clarity. Interestingly, the attentional behaviour switches from decreasing the attentional share of the target, which is in any way higher, to increasing it when the distance between the objects changes.

Overall, the experimental data shows that the active gaze control allows for faster and more accurate action recognition in a large set of conditions. 


\subsection{Comparison with other simulation based active recognition approaches}

This section reports the comparison of the proposed system with several different attention controllers under different levels of gaze dependent noise $\left(\sigma_{d}\right)$.

The attentional models used were*:

Discrim A discriminative model which will be described in the next section.

NoVAC Similarly to the previous experiments, this model does not move gaze during action perception.

Random In this model the attention is randomly allocated to one of the estimated elements position. We must note that the estimated effector position is different for each target hypotheses. Thus, the gaze target is selected between a number of configurations equal to two times the number of targets, one for the target and one for the effector.

RR Round robin allocation of attention. The rotation select first the hypothesis and then selects between predicted target and effector position as gaze targets. So the attentional system alternate gaze toward the objects and gazes toward the effector.

MaxEff This model selects as gaze target the effector position predicted by the model with maximum confidence.

Entropy This is the proposed model where attention is allocated on the basis of the confidence and entropy following the equation 6 . It focuses attention on the most uncertain element of the most probable hypotheses.

The comparison was executed running each model for 1000 trials with 8 objects at medium distance with an attention controller without delays (like in the Ideal VAC case of the previous section). The results are reported in figure 15 in terms of the predictive recognition index (equation 7 ).

All attentional models, included the Random, focus on the estimated positions of the elements. Therefore, all models have the chance of getting low noise observations of the elements during an action. The NoVAC attention focus, instead, is fixed during action execution and positioned near the initial position of one of the elements. The NoVAC will thus perceive some of the elements with an higher noise level during the whole action duration.

When the position dependent noise standard deviation is very low $\left(\sigma_{d}=0.01\right)$ all models have similar performance with the exception of Discrim, which will be discussed in the next section. With a medium level of noise $\left(\sigma_{d}=0.1-0.4\right)$ the proposed attention model Entropy shows better performance than the other models. Between the other models MaxEff has the highest performance. This is likely due to the fact that the Entropy model does not lose time focusing on targets which will not actually participate in the execution of the action. MaxEff is a safe strategy which focuses on the effector instead of looking predictively for the target. The best Entropy model

\footnotetext{
* All the systems used the same predictive model for the estimation of the effector trajectory and
} computation of confidence. 
improves over MaxEff by executing anticipative saccades to reduce the uncertainty of the most probable target position, this in turn allows both better predictions of the effector trajectory and better evaluation of the confidence. With very high noise levels $\left(\sigma_{d}=0.8-1.6\right)$ none of the models achieves satisfying performance. At the highest level of noise the NoVAC model has slightly higher performance than the other systems. This is probably due to the attentional system getting focused on positions far from the real elements due to the noisy estimations. However such a high noise level is not of central for our study and is reported only to illustrate the asymptotic behaviour of the system.

\subsection{Comparison with a discriminative action perception model}

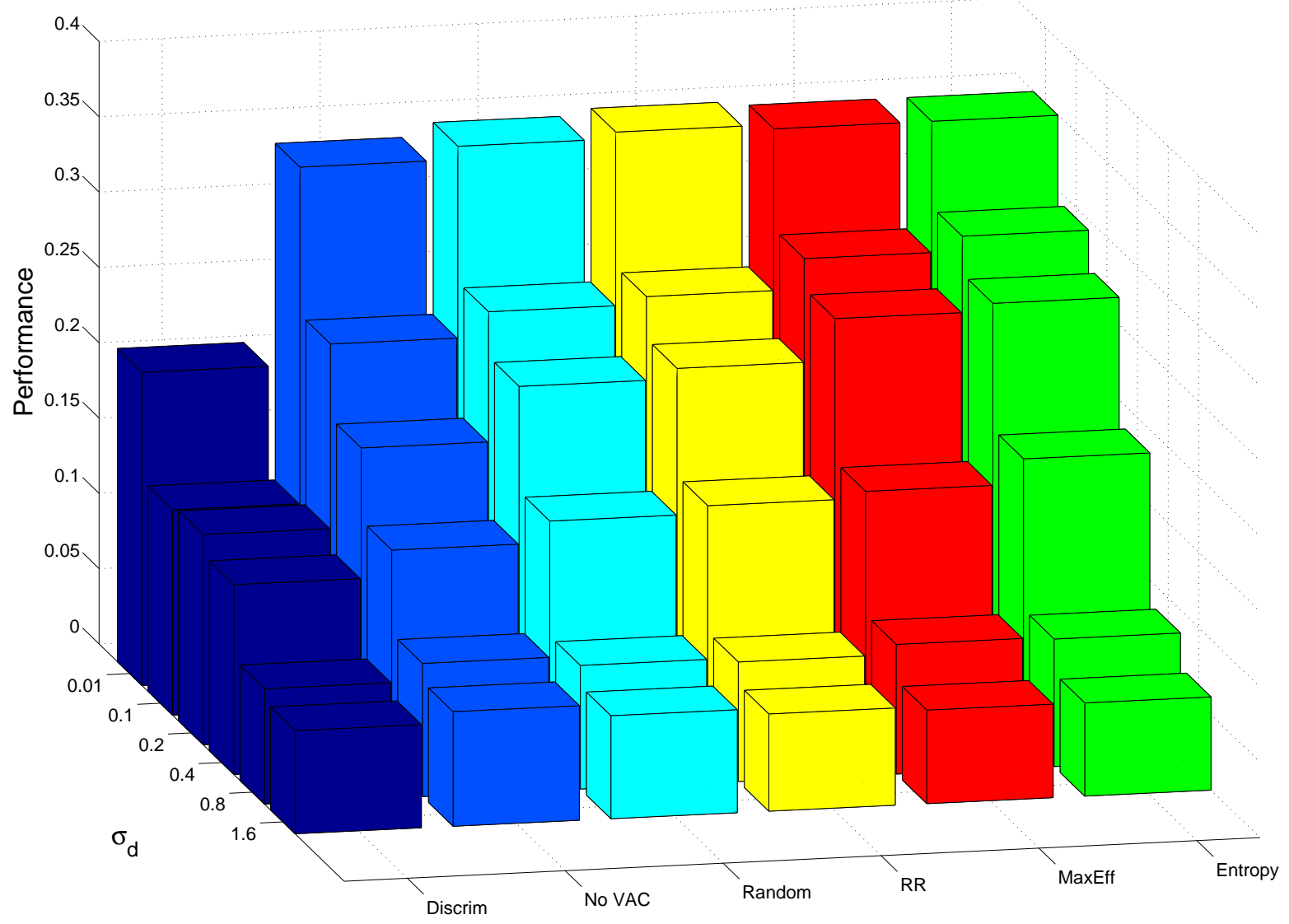

Figure 15. Performance, computed as the predictive recognition index, with different level of gaze dependent noise $\sigma_{d}$ and different gaze target selection policies: (1)Discrim $=$ Discriminative (2)NoVAC $=$ No Visual Attention Control (3) Random $=$ Random target selection (4) $\mathbf{R R}=$ Round Robin (5) MaxEff $=$ Predicted effector position by the maximum confidence model (5) Entropy = Proposed entropy based attention control. The gaze motion is supposed to be ideal (no delays or errors in gaze displacements).

This section reports the comparison between the proposed model, which predicts the next state using the agent own internal motor models, with a discriminative approach to action perception, in which the next state is not predicted using an action and context 
aware model. The hypothesis is that the richer informational context of the proposed model offers higher robustness to observation noise. However, for a fairer comparison in presence of gaze dependent noise, the discriminative model was endowed with Kalman Filters to track the relevant elements of the environment. The difference with the proposed model is that, for the prediction step, a simple brownian motion is assumed for the Kalman Filters associated with the performer's effectors. Thus, the estimated positions are not changed during the update and only the covariance is increased $\sharp$. As usual, the estimations are corrected using the observations in the update step following the prediction step.

The confidence in the discriminative model is computed according to the following rule:

$$
c_{t+1}=c_{t}+\left\|\tilde{\mathbf{p}}_{h}^{t+1}-\tilde{\mathbf{p}}_{g}^{t+1}\right\|-\left\|\tilde{\mathbf{p}}_{h}^{t}-\tilde{\mathbf{p}}_{g}^{t}\right\|
$$

Where $\tilde{\mathbf{p}}_{h}^{t+1}$ is the estimated effector position and $\tilde{\mathbf{p}}_{g}^{t+1}$ is the estimated target position.

In this discriminative model the gaze follows the estimated effector during action execution.

In figure 15 the performance of the discriminative model in terms of predictive recognition index with different levels of gaze dependent noise $\sigma_{d}$ are displayed. The performance are actually lower than those of the No VAC model in all conditions. This lower performance are not only present with the high position dependent noise condition, but also in the very low noise conditions, where only the fixed noise is present $\left(\sigma_{f}\right)$. This can be explained by the limits in distinguishing the actual effector motion from the observation noise and the consequential delay in the update of the estimated effector position. Thus in noisy conditions even with an active perception policy the tested discriminative model is less effective than the predictive one.

\section{Conclusions}

The results reported in this work show that the proposed approach is viable for the problem of action recognition in unknown environments. A relevant contribution given by this paper is related to the importance of using the simulation approach when recognising actions with limited perception. Differently from the teleological and associative approaches, the simulation approach describes mechanisms that implement action recognition by producing dynamic internal representations that can be used also to direct attention. This is particularly apparent in our model, which actually employs the simulated position to drive attention, whilst in previous models prediction was used to modulate bottom-up attention through feature selection (see e.g. [6]). Experimental

\# The brownian motion is modelled, by definition, using an identity transition matrix and a scalar process noise matrix with factor $\sigma_{p}$. For the discriminative model $\sigma_{p}$ was optimised over a wide set of values $\left[2.5 * 10^{-4}, 1.2\right]$ separately for every condition. The discriminative model actually differs from the proposed one only by missing the motor model in the prediction step. In fact, the proposed model has the same process noise model, too. However, in the proposed predictive models optimisation affected the performance only marginally and was thus executed in a smaller search space $\left[2.5 * 10^{-4}, 4 * 10^{-3}\right]$. 
results have confirmed the advantages of the attention-based action recognition system, both in terms of precision and in terms of decision time. This latter aspect is indeed critical when an agent has to interpret or recognise a partner's action. For a robot, being able to understand what a human partner is doing a fraction of a second earlier can be of fundamental importance in order to achieve a meaningful interaction, obviating the need for the human to wait for the robot to interpret her movements.

We are now working on the recognition of real human actions, and the comparison with human performance in the same task. We aim to substitute the current action models with more realistic ones that account for more peculiarities of human movements, that can thus allow for higher accuracy and faster recognition, i.e. using the preshaping of hand for gasping. Then, we plan to test the robustness of the system with respect to incorrect models, and to the presence of a high number of action hypotheses, differentiated also for the different parameters chosen, e.g. different execution speeds. Another interesting improvement would be the use non-myopic target selection, for a better estimation of the relative importance of targets and effectors in visual perception of actions and to account for time consuming gazing movements toward far targets.

While so far we have only been using end-effector position and object positions in the environment, the use of a simulation-theory approach could exploit other relevant sources of information, like the shape of the hand, to improve prediction and recognition of actions. However, the focus of this paper is on selective spatial attention, for which it is crucial to predict when and where will information be available. Obviously, using other kinds of information will change informational contributions available in the different positions. The prediction of the positions of information sources and their selection will still be essential for any attentional policy using additional information sources. Extending our approach to such conditions is currently being investigated.

An interesting insight emerging from our work concerns the presence of an exploratory behaviour before attention can actually focus only on the hand and the actual target of the action (e.g., see figure 6 or figure 14 with a more complex attentional behaviour). While our model shows predictive saccades similar to those found by Flanagan and Johansson [29], in their experiments there was no exploratory behaviour, and they suggested that their experimental results supported the hypothesis that humans reuse the attentional plans employed during action execution for action perception. The strong predictive attentional behaviour shown in their experiment can also result from the presence of a very low level of uncertainty in the observed task, where only one possible and known action goal was present at each moment.

In the setup of our experiments instead, the uncertainty of the targets of the actions is higher. In such setup, which is arguably more common in normal social interactions, directly reusing the same attentional plans used during action execution would need to be complemented by an initial exploratory phase which reduces uncertainty. Given the different nature of these two phases, they are also probably implemented by two different mechanisms. However, the model proposed in this paper, while perfectly consistent with the simulation theory of action perception, does not need two different mechanisms to 
produce an exploratory phase and a predictive phase, but only the online estimation of the informativeness of the different possible gaze targets. If experimental results confirm the presence of such exploratory attentional behaviour, it will be interesting to understand which of the two theories, if any, is actually implemented by the brain.

\section{Acknowledgments}

This research has received funding from the European Union Seventh Framework Programme FP7/2007-2013, under grant agreement No [270490]- [EFAA].

\section{References}

[1] R. Bajcsy. Active perception. Proceedings of the IEEE, 76(8):966-1005, 1988.

[2] D.H. Ballard. Animate vision. AI, 48:57-86, 1991.

[3] G.C.H.E. de Croon, E.O. Postma, and H.J. van den Herik. Adaptive gaze control for object detection. Cognitive Computation, 3(1):264-278, 2011.

[4] Y. Demiris and B. Khadhouri. Hierarchical attentive multiple models for execution and recognition of actions. Robotics and Autonomous Systems, 54:361-369, 2006.

[5] Y. Demiris and B. Khadhouri. Content-based control of goal-directed attention during human action perception. Journal of Interaction Studies, 9(2):353-376, 2008.

[6] Yiannis Demiris and Gavin Simmons. Perceiving the unusual: temporal properties of hierarchical motor representations for action perception. Neural Networks, 19(3):272-284, Apr 2006.

[7] T. Grent and M.G. Woldorff. Timing and sequence of brain activity in top-down control of visualspatial attention. PLoS biology, 5(1):e12, 2007.

[8] L. Itti and C. Koch. Computational modelling of visual attention. Nat Rev Neurosci, 2(3):194-203, Mar 2001.

[9] K. Kastella. Discrimination gain to optimize detection and classification. IEEE Transactions on Systems, Man and Cybernetics, Part A: Systems and Humans, 27(1):112-116, 1997.

[10] Cody Kwok and Dieter Fox. Reinforcement learning for sensing strategies. In IEEE/RSJ International Conference on Intelligent Robots and Systems (IROS2004), 2004.

[11] Michael F Land. Eye movements and the control of actions in everyday life. Prog Retin Eye Res, 25(3):296-324, 2006.

[12] David Marr. Vision: A Computational Investigation into the Human Representation and Processing of Visual Information. W. H. Freeman, New York, 1982.

[13] D. Ognibene, C. Balkenius, and G. Baldassarre. Integrating epistemic action (active vision) and pragmatic action (reaching): A neural architecture for camera-arm robots. In Proceedings of the Tenth International Conference on the Simulation of Adaptive behaviour, 2008.

[14] D. Ognibene, Y. Wu, K. Lee, and Y. Demiris. Hierarchies for embodied action perception. Under review, 2012.

[15] Dimitri Ognibene, G. Pezzulo, and G. Baldassarre. How can bottom-up information shape learning of top-down attention control skills? In Proceedings of 9th International Conference on Development and Learning, 2010.

[16] Constantin A. Rothkopf, Dana H. Ballard, and Mary M. Hayhoe. Task and context determine where you look. Journal of Vision, 7(14):16.1-1620, 2007.

[17] Uta Sailer, J. Randall Flanagan, and Roland S Johansson. Eye-hand coordination during learning of a novel visuomotor task. J Neurosci, 25(39):8833-8842, 2005.

[18] M. Sarabia, R. Ros, and Y. Demiris. Towards an open-source social middleware for humanoid robots. In Proc. 11th IEEE-RAS Int Humanoid Robots (Humanoids) Conf, pages 670-675, 2011. 
[19] Jürgen Schmidhuber and Rudolf Huber. Learning to generate artificial fovea trajectories for target detection. Int J Neural Syst, 2(1-2):135-141, 1991.

[20] Eric Sommerlade and Ian Reid. Information theoretic active scene exploration. In Proc. IEEE Computer Vision and Pattern Recognition (CVPR), May 2008.

[21] Mototaka Suzuki and Dario Floreano. Enactive robot vision. Adapt Behav, 16(2-3):122-128, 2008.

[22] Jochen Triesch, Dana H Ballard, Mary M Hayhoe, and Brian T Sullivan. What you see is what you need. J Vis, 3(1):86-94, 2003.

[23] E. Vul, M. C. Frank, J. B. Tenenbaum, and G. Alvarez. Explaining human multiple object tracking as resource-constrained. approximate inference in a dynamic probabilistic model. In Advances in Neural Information Processing Systems, volume 21, 2009.

[24] Alfred L. Yarbus. Eye movements and vision. Plenum Press, 1967.

[25] G. Pezzulo, and H. Dindo. What should I do next? Using shared representations to solve interaction problems. Experimental Brain Research, 1-18, 2011.

[26] C. L.Baker, R. Saxe, and J. B. Tenenbaum. Action understanding as inverse planning. Cognition, 113(3):329349, 2009.

[27] G. Pezzulo, and D. Ognibene. Proactive Action Preparation: Seeing Action Preparation as a Continuous and Proactive Process. Motor control, 16(3):386-424, 2012.

[28] B. W. Tatler, M. M. Hayhoe, M. F. Land, and D. Ballard. Eye guidance in natural vision: Reinterpreting salience $J$ Vis, 11, 1-23 2011.

[29] J. R. Flanagan and R. S. Johansson. Action plans used in action observation Nature, 424:769-771, 2003.

[30] Ugo Pattacini. Modular Cartesian Controllers for Humanoid Robots: Design and Implementation on the iCub. Istituto Italiano di Tecnologia, 2010.

[31] V. Tikhanoff, A. Cangelosi, P. Fitzpatrick, G. Metta, L. Natale, F. Nori. An open-source simulator for cognitive robotics research: the prototype of the iCub humanoid robot simulator In Proc. 8th Workshop on Performance Metrics for Intelligent Systems, 57:61, 2008.

[32] A. Andreopoulos, and J. K. Tsotsos. A computational Learning Theory of Active Object Recognition Under Uncertainty. Int. J. Comp. Vis. 101(1):95-142, 2013 